\title{
Assessment of the Effect of Autograft Orientation on Peripheral Nerve Regeneration Using Diffusion Tensor Imaging
}

\author{
Ashkan Afshari, MD ${ }^{\star}$, , Lyly Nguyen, MD ${ }^{\star}, \ddagger$, Nathaniel D. Kelm, BS $§, \|$, Justine S. Kim, BS ${ }^{\star}$, \\ Nancy L. Cardwell, BS ${ }^{\star}$, Alonda C. Pollins, MS ${ }^{\star}$, Ravinder Bamba, MD ${ }^{\star}$, R. Bruce Shack, \\ MD*, Mark D. Does, PhD§,\|, and Wesley P. Thayer, MD, PhD* \\ *Department of Plastic Surgery, Vanderbilt University Medical Center, Nashville, TN \\ tDepartment of Surgery, University of South Carolina, Columbia, SC \\ ‡Department of Surgery, Morristown Medical Center, Morristown, NJ \\ $\S$ Department of Biomedical Engineering, Vanderbilt University Medical Center, Nashville, TN \\ IIInstitute of Imaging Science, Vanderbilt University Medical Center, Nashville, TN
}

\begin{abstract}
Purpose-Given no definite consensus on the accepted autograft orientation during peripheral nerve injury repair, we compare outcomes between reverse and normally oriented autografts using an advanced magnetic resonance imaging technique, diffusion tensor imaging.
\end{abstract}

Methods-Thirty-six female Sprague-Dawley rats were divided into 3 groups: sham—left sciatic nerve isolation without injury, reverse autograft-10-mm cut left sciatic nerve segment reoriented $180^{\circ}$ and used to coapt the proximal and distal stumps, or normally oriented autograft-10-mm cut nerve segment kept in its normal orientation for coaptation. Animals underwent sciatic functional index and foot fault behavior studies at 72 hours, and then weekly. At 6 weeks, axons proximal, within, and distal to the autograft were evaluated using diffusion tensor imaging and choline acetyltransferase motor staining for immunohistochemistry. Toluidine blue staining of $1-\mu \mathrm{m}$ sections was used to assess axon count, density, and diameter. Bilateral gastrocnemius/soleus muscle weights were compared to obtain a net wet weight. Comparison of the groups was performed using Mann-Whiney $U$ or Kruskal-Wallis $H$ tests to determine significance.

\begin{abstract}
Results-Diffusion tensor imaging findings including fractional anisotropy, radial diffusivity, and axial diffusivity were similar between reverse and normally oriented autografts. Diffusion tensor imaging tractography demonstrated proximodistal nerve regeneration in both autograft groups. Motor axon counts proximal, within, and distal to the autografts were similar. Likewise, axon count, density, and diameter were similar between the autograft groups. Muscle net weight at 6 weeks and behavioral outcomes (sciatic functional index and foot fault) at any tested time point were also similar between reverse and normally oriented autografts.
\end{abstract}

Reprints: Ashkan Afshari, MD, Department of Plastic Surgery, Vanderbilt University Medical Center, Medical Center North, 1161

21st Avenue S, S2221, Nashville, 37232 TN. ashkan.afshari83@gmail.com.

Ashkan Afshari and Lyly Nguyen contributed equally to this manuscript and are co-first authors.

Conflicts of interest and sources of funding: none declared. 
Conclusions-Diffusion tensor imaging may be a useful assessment tool for peripheral nerve regeneration. Reversing nerve autograft polarity did not demonstrate to have an influence on functional or regenerative outcomes.

\section{Keywords}

autograft; diffusion tensor imaging; orientation; polarity; peripheral nerve regeneration; DTI

Autologous nerve grafting is considered the gold standard to bridge nerve gaps. Autograft orientation, reversed or normal, has previously been studied to determine the effects of polarity on nerve regeneration. In both histological and functional indices, studies have demonstrated no differences found with the orientation of nonbranched autografts. ${ }^{1-3}$ Yet, an animal study by Ansselin and Davey ${ }^{4,5}$ demonstrated a greater cross-sectional area and, perhaps, improved axonal regeneration at the distal stump with reversed autografts with branch points. Among the few published studies on this topic, each uses a limited number of assessment tools to compare outcomes between reverse and normally oriented autografts. Therefore, there is uncertainty with respect to the best nerve autograft orientation during peripheral nerve repair.

Recently, more advanced methods of determining nerve growth have been developed. Diffusion tensor imaging (DTI) is a magnetic resonance imaging method that has the ability to noninvasively assess axonal integrity and regeneration in peripheral nerves objectively and visually. ${ }^{6,7}$ Diffusion tensor imaging parameters such as fractional anisotropy (FA), axial diffusivity (AD), and radial diffusivity (RD) reflect the anisotropic diffusion of water molecules along and perpendicular to axon bundles, which are affected by axon density and myelin thickness. ${ }^{8}$ Values obtained from DTI aid in assessing nerve regeneration and degeneration. Furthermore, DTI allows visualization of neural tracts and assessment of changes in axon microstructure. ${ }^{6-8}$ Using this emerging technology in addition to traditional assessment tools, this study provides a comprehensive comparison of reverse and normally oriented autograft repairs in a nonbranched model.

\section{METHODS}

\section{Experimental Design}

This study was performed using a rat sciatic nerve model using a total of 36 animals divided into 3 groups: reverse autografts $(n=12)$, normally oriented autografts $(n=12)$, and shams $(\mathrm{n}=12)$ to serve as a control. Postoperatively, all rats underwent behavioral studies at 72 hours, and then weekly. At 6 weeks, the left sciatic nerve was harvested; half were evaluated using (1) immunohistochemical staining of 5- $\mu$ m-thick sections and toluidine blue staining of 1- $\mu$ m-thick sections or (2) DTI at segments proximal, within, and distal to the autografts. Only 1 segment was evaluated in the sham group. Bilateral gastrocnemius/soleus muscles were harvested to obtain net wet weights to assess the degree of muscle atrophy.

\section{Animal Model}

Adult female Sprague-Dawley rats (200-250 g; Charles River Lab) were used in the experiment. Animals were housed in a central animal care facility and provided food and 
water ad libitum. All experimental procedures were approved and performed in accordance with the standards of our institutional animal care and use committee.

\section{Surgical Model}

Animals were anesthetized via inhaled isoflurane (2\%). The left hindquarter was shaved and prepped in a sterile fashion. A 3-cm incision was made from the left sciatic notch and extended inferiorly, parallel and just posterior to the left femur. The biceps femoris was reflected to expose the sciatic nerve. The nerve was trimmed free of any perineural tissue for all groups. For the autograft groups, a 10-mm nerve gap was created in the mid-portion of the sciatic nerve with microsurgical scissors. The transected portion of the nerve was either coapted to the sciatic nerve in normal orientation or coapted after reversing the cut segment $180^{\circ}$ proximodistally. An end-to-end repair using 4 equidistant 9-0 nylon sutures (Ethicon, Somerville, NJ) around each end of the autograft was performed using standard microsurgical technique under a Carl Zeiss Stemi Sv-11 microscope (Thornwood, NY). For all groups, the muscle was imbricated to cover the nerve using 5-0 monocryl in a horizontal mattress fashion. The skin was closed in a running subcuticular fashion with 5-0 monocryl suture.

\section{Behavioral Testing}

Before surgery, animals underwent habituation trials for acclimation purposes. Postoperatively, foot fault (FF) asymmetry and sciatic functional index (SFI) test were performed at 72 hours followed by weekly intervals, up to 6 weeks. To prevent possible bias, behaviors testers were blinded to the surgical protocols of each animal.

\section{Foot Fault}

Animals were allowed to roam freely on a wire mesh grid measuring $45 \times 30 \mathrm{~cm}$, with square openings measuring $2.5 \times 2.5 \mathrm{~cm}$, and elevated $2 \mathrm{~cm}$ above a solid base. Trials of 50 total steps for each hind limb were recorded for each animal. A full fault was recorded if the animal's hind limb fell through the grid opening and touched the base. A partial fault was recorded if the animal's hind limb fell through the opening but did not touch the base. An FF asymmetry score was calculated using the following equation: composite FF score $=(\mathrm{n}$ partial faults $\times 1)+(\mathrm{n}$ full faults $\times 2), \% \mathrm{FF}=($ composite $\mathrm{FF}$ score/total number of steps $) \times$ $100 \%$, FFasymmetry score $=\% \mathrm{FF}$ (normal hind limb $)-\% \mathrm{FF}$ (surgical hind limb).

\section{Sciatic Functional Index}

The hind limbs were inked, such that red designated the unoperated limb and black designated the surgical limb. Animals were placed on the beam farthest from the cage, and 6 consecutive ( 3 from each limb) foot prints were recorded. As previously published, ${ }^{9-11}$ the SFI was scored using the following parameters: normal print length (NPL), normal toe spread (NTS), normal intermediary toe spread (NIT), experimental print length (EPL), experimental toe spread (ETS), and experimental intermediary toe spread (EIT). Sciatic functional index scores were calculated using the following formula: SFI $=-38.3$ ([EPL NPL]/NPL $)+109.5([$ ETS - NTS $] /$ NTS $)+13.3\left([\right.$ EIT - NIT]/NIT $)-8.8 .{ }^{12}$ 


\section{Sciatic Nerve and Gastrocnemius/Soleus Muscle Harvest}

Once the animals reached the 6-week end point, they were euthanized with Euthasol (Virbac, Fort Worth, Tex) intracardiac injections. The prior incision was reopened, and the full length of the nerve was harvested at the most proximal and distal points for histological or DTI analysis. The gastrocnemius/soleus muscles from the bilateral hind limbs were then carefully dissected off surrounding tissues and weighed (gm) to obtain a net wet weight (normal hind limb muscle weight - surgical hind limb muscle weight).

\section{Histology}

Immunohistochemistry-Immunohistochemical staining was performed using commercial antibodies specifically directed against choline acetyltransferase (Millipore, Temecula, Calif ) for motor axons at 1:200 for 1 hour as previously described. ${ }^{13}$ Digital images of slides were acquired with an Olympus C-35AD-4 microscope at 10x using ImagePro Plus 7.0 (Media Cybernetics, Bethesda, Md). Stained axons were counted using ImageJ (Wayne Rasband, National Institute of Mental Health, Bethesda, Md) software.

Toludine Blue Stain-Harvested nerves were fixed in 2\% paraformaldehyde/3\% glutaraldehyde, postfixed with $1 \% \mathrm{OsO}_{4}$ solution, dehydrated in increasing concentrations of ethanol, and then embedded in resin at $60^{\circ}$ for 48 hours. Samples were cut into $1-\mu \mathrm{m}$-thick sections and stained with $1 \%$ toluidine blue. Digital images of slides were acquired with an Olympus C-35AD-4 microscope. Axon count, density (number $/ \mathrm{mm}^{2}$ ), and diameter $(\mu \mathrm{m})$ were measured using Image-Pro Plus 7.0. Within each segment of each nerve, axon density was calculated by manually counting axons within 3 randomly selected areas at $40 \times$ and then averaged to avoid selection bias. Total axon count was calculated by multiplying the average axon density by the nerve cross-sectional area. Axon diameter was calculated using the average diameter of 60 randomly chosen axons.

\section{Diffusion Tensor Imaging}

Preparation-Harvested nerves were incubated in 4\% glutaraldehyde/ $0.5 \%$ paraformaldehyde in phosphate-buffered saline at $4{ }^{\circ} \mathrm{C}$. After 24 hours postfixation, excisednerveswereplacedinphosphate-bufferedsaline+2mM Gd-DTPA (Magnevist, Bayer HealthCare, Wayne, $\mathrm{NJ}$ ) at $4^{\circ} \mathrm{C}$ for at least 24 hours before imaging. For imaging, excised nerves were placed in 2-mm outer diameter glass capillary tubes filled with a perfluropolyether liquid (Fomblin, Solvay Solexis, Thorofare, NJ) for susceptibility matching, preventing tissue dehydration, and a signal-free background. For higher throughput, 6 excised sciatic nerves in a hexagonal arrangement were imaged simultaneously.

Diffusion Tensor Imaging Acquisition-Magnetic resonance imaging was performed on a 4.7-T 31-cm horizontal bore Agilent DirectDrive scanner (Agilent Technologies, Santa Clara, Calif) using a 38-mm Litz quadrature coil (Doty Scientific, Columbia, SC) for radiofrequency transmission and reception. Diffusion tensor imaging data were acquired using a 3-dimensional (3D) diffusion-weighted spin echo sequence with repetition time/echo time $=170 / 23.0 \mathrm{~ms}, 12$ signal averages, field-of-view $=9.6 \times 9.6 \times 14.4 \mathrm{~mm}^{3}$, and matrix size $=96 \times 96 \times 32$ for a nominal resolution of $100 \times 100 \times 450 \mu \mathrm{m}^{3}(450 \mu \mathrm{m}$ along the 
nerve). Diffusion weighting was achieved with $\delta / \Delta=4 / 12 \mathrm{~ms}$, a prescribed $b$ value of 2000 $\mathrm{s} / \mathrm{mm}^{2}$, and 6 directions. One $b=0$ image was acquired for a total of 7 images in a scan time of approximately 12 hours.

Diffusion tensor imaging Analysis-Image data reconstruction was performed using in-house written code in MATLAB (MathWorks, Natick, Mass), and DTI analysis was implemented in ExploreDTI. ${ }^{14}$ 3D image volumes were zero padded 2 times in each direction during reconstruction from k-space data. Diffusion tensors were estimated voxelwise using a linear least-squares approach. From the diffusion tensor, DTI metrics including FA, AD, and RD were computed voxel-wise and DTI tractography was performed. Then, DTI parameters were measured for 3 regions of interest (ROIs): $1.5 \mathrm{~mm}$ proximal to the proximal stump, the middle of the autograft, and $1.5 \mathrm{~mm}$ distal to the distal stump.

\section{Statistical Analysis}

All analyses were performed using IBM SPSS Statistics 23.0 software (IBM Corp, Armonk, NY). Statistical significance was determined using nonparametric statistics, Mann-Whitney $U$, or Kruskal-Wallis $H$ test. Values are reported as median [interquartile range (IQR)]. Statistical significant was assigned a $P$ value of less than 0.05 .

\section{RESULTS}

\section{Behavioral Evaluations of Sciatic Nerve Function}

At all test time points, reverse and normally oriented autografts demonstrated similar values in both FF and SFI scores. The sham group, however, consistently demonstrated noninjured behavioral patterns compared with both autograft groups (Fig. 1A, B).

\section{Gastrocnemius/Soleus Net Wet Weights}

Muscle net wet weights in reverse and normally oriented autografts did not differ [1.36 g (IQR, 1.30-1.44) vs $1.33 \mathrm{~g}$ (IQR, 1.24-1.37); $P=0.68$ ), although were significantly more atrophied than shams $(0.02 \mathrm{~g}$; IQR, -0.7 to $0.03 ; P<0.05)$.

\section{Nerve Histology With Immunohistochemistry}

After staining with choline acetyltransferase for motor axons, cross sections proximal to, within, and distal to the graft were evaluated. Animals demonstrated no difference in motor axon counts at each segment between reverse and normally oriented autografts. Figures 2 and 3 demonstrate motor axon counts and representative slides within each group at any given nerve segment, respectively.

\section{Nerve Histology With Toludine Blue Axon Staining}

We observed morphologically normal axons with interspersed regenerative patterns and edema, $1.5 \mathrm{~mm}$ proximal to the proximal injury site in both autograft groups. The total number of axons within the grafts in the reverse autograft group was 8429 (IQR, 82748762 ), and 8630 (IQR, 6353-8982) axons in the normally oriented autograft group ( $P=$ 0.57). Axon density was 7429 axons $/ \mathrm{mm}^{2}$ (IQR, 7291-7722) in the reverse group, with a density of 7739 axons $/ \mathrm{mm}^{2}$ (IQR, 7470-7951) in normally oriented autografts $(P=0.57)$. 
Axon diameter was $5.09 \mu \mathrm{m}$ (IQR, 4.64-5.52) versus $5.16 \mu \mathrm{m}$ (IQR, 4.97-5.83), respectively $(P=1.00)$. Distally along the length of the nerve in both autograft groups, axon density decreased as did axon diameter. In the distal segment, the axon count was 7668 (IQR, 7010$7773)$ and 7547 (IQR, 6715-7836) $(P=1.00)$ in the reverse and normal autografts, respectively. Axon density was 5905 axons $/ \mathrm{mm}^{2}$ (IQR, 5398-5985) in reverse autografts, with an axon density of 5812 axons $/ \mathrm{mm}^{2}$ (IQR, 5171-6034) in the normally oriented autografts $(P=1.00)$. The diameter of axons were $3.92 \mu \mathrm{m}$ (IQR, 3.67-4.16) in the reverse autograft group and $3.51 \mu \mathrm{m}$ (IQR, 3.20-4.19) in the normally oriented group $(P=1.00)$. Shams had an axon count of 6778 (IQR, 6197-7212), density of 6655 axons $/ \mathrm{mm}^{2}$ (IQR, 6084-7081), and diameter of $8.74 \mu \mathrm{m}$ (IQR, 8.45-9.17). Representative images are seen in Figure 4.

\section{Diffusion Tensor Imaging and Tractography}

Fractional anisotropy, AD, and RD were measured for each ROI. Diffusion tensor imaging measures comparing reverse and normally oriented autografts were comparable, signifying similar axonal regeneration. As such, there was no statistically significant difference in FA, $\mathrm{AD}$, and $\mathrm{RD}$ at any segment when comparing reverse and normally oriented autograft to each other. When comparing shams to autografts, both reverse and normally oriented autografts demonstrated a statistically significant decrease in FA at ROIs within and distal to the graft compared with shams. This decrease in FAwas also evident at the proximal segment of the autografts in comparison to shams, which corresponded to edematous findings on toluidine blue histological analysis. Axial diffusivity was similar, although RD was significantly increased in the autograft groups compared with shams. See Table 1 for a complete comparison of DTI values between sham and autograft groups. Diffusion tensor imaging tractography displayed proximodistal regenerative tracts in both reverse and normally oriented autograft groups (Fig. 5).

\section{DISCUSSION}

Few experiments have been performed to explore the effect of autograft orientation during peripheral nerve repair, with the most recent being more than a decade ago. Each study has been typically limited to very few assessment tools to compare reverse and normally oriented autografts. Furthermore, results between different studies have been inconsistent, therefore leading to ambiguity in the proper autograft orientation that would result in the best regenerative outcome. Stromberg et a ${ }^{1}$ performed the first study in a rat model to assess nerve autograft polarity in 1979. Their study demonstrated outcomes were independent of polarity of the nerve graft. Nerve conduction velocity and amplitude, as well as histological analyses, were indistinguishable between reverse and normally oriented autografts at 6 months. Similar findings were demonstrated in a rabbit model, using 2 -cm cable grafts. ${ }^{3}$ In our study, histological analysis at 6 weeks, whether by immunohistochemistry or toluidine blue staining, likewise demonstrated similarity in quantitative axonal measures such as axon count, density, and diameter in reverse and normally oriented autografts. Therefore, both short- and long-term axonal regeneration appear to be similar irrespective of nerve autograft polarity. Concordant with equivalent axonal growth, in our study a comparable degree of muscle atrophy was observed in the affected limb between the autograft groups, which was 
also evident in a 6-month study by Nakatsuka et $\mathrm{al}^{3}$ in a rabbit model. Ansselin and Davey, in their rat experiment using fibularis or tibialis nerves, found that nerve regeneration within the autograft was similar using either orientation; however, the distal cross-sectional area in the normally oriented autografts was reduced. They observed that axonal regeneration occurs through terminal nerve branch points in the normally oriented autografts, whereas they "disappear" in reverse autografts. From these findings, they concluded that some axonal regeneration is lost distally through terminal branches in normal grafts. ${ }^{4,5}$ The studies by Ansselin and Davey only provided histological analysis, without offering other assessments such as animal behavior, but nonetheless have been the cornerstone of why some advocate reversing the orientation when using autografts with branch points.

Sotereanos et $\mathrm{al}^{2}$ studied nerve polarity in a nonbranched rat sciatic nerve model using the Bain modification of de Medinaceli's SFI behavioral study to assess functional outcomes. Functional recovery and histological analysis of regeneration were found to be equivalent regardless of autograft orientation. Dellon and Mackinnon ${ }^{15}$ have shown SFI to be a very useful tool after nerve injury. The similar weekly SFIs demonstrated up to 6 weeks in our study, and up to 120 days in Sotereanos et al study evaluating SFI between reverse and normally oriented autografts, suggest similar short- and long-term functional outcomes. ${ }^{2}$ Foot fault, a behavioral injury assessment model described for sensorimotor recovery, particularly for up to 30 to 60 days postinjury, ${ }^{16}$ also showed similar recovery patterns up to 6 weeks.

The uncertainty regarding the best autograft orientation is evident due to discrepancies in findings between studies by Ansselin and Davey in branched models with other published literature. Furthermore, despite some evidence of similar outcomes between reverse and normally oriented autograft in nonbranched models, proper autograft orientation remains ambiguous. Some advocate the use of reverse autografts due to the concerns of losing axons through major branches, ${ }^{4,5,17}$ whereas some advocate keeping the normal physiologic orientation to either to maintain physiologic axoplasmic flow ${ }^{18}$ or because reverse autografts do not overcome the branch point problem. ${ }^{19}$ Others either identify that there is no difference ${ }^{1-3}$ or do not make mention of a suggested orientation.

In this study, we used DTI to assess axonal regeneration. Previous studies have evaluated peripheral nerves with DTI metrics and compared them to histological findings and animal behavior scoring. ${ }^{20-22}$ Lehmann et $\mathrm{al}^{6}$ found that there was a positive correlation between FA value and the total number of myelinated axons after crush injury in the mouse model. Various other studies have had similar results, validating DTI as an appropriate tool in imaging peripheral nerve injury models. ${ }^{6,20-22}$ This advanced magnetic resonance imaging technology characterizes and quantifies diffusion of water in a tissue of interest (ie, nerves) and reflect alterations in tissue microstructure. Isotropic diffusion refers to diffusion that is equal in all directions, as would be seen in a 3D sphere. However, nerve tissue is constrained by myelinated fibers and fascicles, causing diffusion of water along a dominant pathway, termed anisotropic diffusion. Typically, AD and RD are used to derive quantitative DTI parameters. Axial diffusivity refers to the extent of water molecules parallel to the direction of nerve fibers, whereas RD is the extent of diffusion of water molecules perpendicular to the direction of nerve fibers. The values are used to compute FA, an index of how different 
the principal diffusions are, with values ranging from 0 to 1 . A value closer to 0 corresponds to random, multidirectional diffusion of water, whereas a value closer to 1 indicates that the nerve is directing diffusion strictly along one direction in its axis (anisotropy) ${ }^{6}$ The FA for a normal, uninjured nerve is expected to be between 0.6 and 0.8 , where axons are aligned relatively linearly, as FA fails to reach the value of 1 due to the presence of structures such as glial cells, axons, Schwann cells, myelin, etc, which can provide a barrier to complete anisotropic diffusion. ${ }^{8,23}$ Nerve injury is associated with less directionality, as, for example, myelin disruption causes unorganized diffusion, thus exhibiting lower values of FA. In this study, FA values for shams were similar to those of other uninjured models in previous studies and were higher than those in the autograft groups. Although some studies have demonstrated that RD is a useful nerve monitoring parameter, most studies agree that changes in FA correlate with peripheral nerve regeneration. ${ }^{8,20,22,24}$ Morisaki et al' ${ }^{22}$ study of crush injury in a rat sciatic nerve model demonstrated decreased FA and increased RD in the degenerative phase up to 3 weeks after injury. This is similar to Yamasaki et al's ${ }^{20}$ study on crush injury of rabbit sciatic nerve where a decreased FA was observed until 4 weeks postinjury, representing lowered anisotropic diffusion due to the loss of nerve integrity. These time points represent the end of Wallerian degeneration and the continued clearance of myelin and axonal debris. At 6 weeks, we observed a higher FA in the autograft groups compared with the findings of Morisaki et al and Yamasaki et al at earlier time points. This indicates that at 6 weeks, the autograft groups have entered the axonal regeneration phase, however incompletely, as FA levels have not reached values of shams. Radial diffusivity was also significantly increased in autograft groups compared with shams, as demonstrated in previous DTI studies, though inconsistently. The comparison of axonal regeneration, however, proved to be similar between the autograft groups, as the degrees of FA, RD, and AD were similar regardless of orientation. Diffusion tensor imaging data in this study comparing autograft to shams, and autografts with each other are consistent with the behavioral and histological results observed in our experiment. Furthermore, our DTI results are comparable to outcomes in previous DTI studies in crush-injured and non-gap nerve injury repair models. These findings suggest that DTI may be a useful tool in evaluating and assessing nerve injury, including in nerve gap models. It is important to note that all studies, including the current study, comparing reverse and normally oriented autografts are in animal models due to the inability to provide histological analysis in human models. Perhaps, DTI may be a useful noninvasive tool to provide objective data in human nerve models.

The results of this study suggest that autograft orientation may have no bearing on functional or histological outcomes for at least 6 weeks postoperatively. This time point was chosen to minimize the effect of enhanced neuroregenerative capacity seen in rat models, as experiments with later time points may artificially result in similar outcomes regardless of the type of treatment. ${ }^{25}$ Furthermore, as DTI measures begin improving after 3 to 4 weeks in crush injury models, our goal was to capture any early difference, as these measures have never been explored in autograft models. Longer term DTI outcomes need to be further explored in future studies.

Although it has been previously described that some regenerating axons may be lost in terminal branches, branches have also been shown to "disappear" or collapse ${ }^{4,5}$; therefore, 
branching in the graft may have minimal effect. Our study does not evaluate the effect of branch points and, therefore, cannot attest to its consequence. Under conditions where nonbranching autografts are used, orientation was not shown to influence functional or regenerative measures. Although branched autografts (ie, sural nerve) are more commonly used than nonbranched, there has been a gain in popularity of allograft use for nerve repairs. Their orientation is usually unknown, and therefore, the results of this study may provide reassurance that outcomes are similar regardless of orientation. Future studies should investigate the outcomes of reverse and normal autografts with branch points using a comprehensive list of assessment tools including DTI, and perhaps in clinically comparable larger sized nerve models, including human models, to investigate whether disappearance or collapse of branches occurs and how this may or may not affect outcomes.

\section{Acknowledgments}

We would like to acknowledge the Animal Care staff and Amy Nunnally for their assistance in animal care, as well as the electron microscopy core lab and Vanderbilt University Institute of Imaging Science for their help with this research.

\section{References}

1. Stromberg BV, Vlastou C, Earle AS. Effect of nerve graft polarity on nerve regeneration and function. J Hand Surg Am. 1979; 4:444-445. [PubMed: 501056]

2. Sotereanos DG, Seaber AV, Urbaniak JR. Reversing nerve-graft polarity in a rat model: the effect on function. J Reconstr Microsurg. 1992; 8:303-307. [PubMed: 1629808]

3. Nakatsuka H, Takamatsu K, Koshimune M, et al. Experimental study of polarity in reversing cable nerve grafts. J Reconstr Microsurg. 2002; 18:509-515. [PubMed: 12177822]

4. Ansselin AD, Davey DF. Axonal regeneration through peripheral nerve grafts: the effect of proximodistal orientation. Microsurgery. 1988; 9:103-113. [PubMed: 3173075]

5. Ansselin AD, Davey DF. The regeneration of axons through normal and reversed peripheral nerve grafts. Restor Neurol Neurosci. 1993; 5:225-240. [PubMed: 21551905]

6. Lehmann HC, Zhang J, Mori S, et al. Diffusion tensor imaging to assess axonal regeneration in peripheral nerves. Exp Neurol. 2010; 223:238-244. [PubMed: 19879260]

7. Sheikh KA. Non-invasive imaging of nerve regeneration. Exp Neurol. 2010; 223:72-76. [PubMed: 19616546]

8. Boyer RB, Kelm ND, Riley DC, et al. 4.7-T diffusion tensor imaging of acute traumatic peripheral nerve injury. Neurosurg Focus. 2015; 39:1-9.

9. De Medinaceli L, Freed WJ, Wyatt RJ. An index of the functional condition of rat sciatic nerve based on measurements made from walking tracks. Exp Neurol. 1982; 77:634-643. [PubMed: 7117467]

10. Sarikcioglu L, Demirel BM, Utuk A. Walking track analysis: an assessment method for functional recovery after sciatic nerve injury in the rat. Folia Morphol (Warsz). 2009; 68:1-7. [PubMed: 19384823]

11. Varejão AS, Meek MF, Ferreira AJ, et al. Functional evaluation of peripheral nerve regeneration in the rat: walking track analysis. J Neurosci Methods. 2001; 108:1-9. [PubMed: 11459612]

12. Bain JK, Mackinnon SE, Hunter DA. Functional evaluation of complete sciatic, peroneal, and posterior tibial nerve lesions in the rat. Plast Reconstr Surg. 1989; 83:129-138. [PubMed: 2909054]

13. Riley DC, Bittner GD, Mikesh MA, et al. Polyethylene glycol-fused allografts produce rapid behavioral recovery after ablation of sciatic nerve segments. J Neurosci Res. 2015; 93:572-583. [PubMed: 25425242]

14. Leemans A, Jeurissen B, Sijbers J, et al. ExploreDTI: a graphical toolbox for processing, analyzing, and visualizing diffusion MR data. Proc Intl Soc Mag Reson Med. 2009; 17:3537. 
15. Dellon AL, MacKinnon SE. Sciatic nerve regeneration in the rat. Validity of walking track assessment in the presence of chronic contractures. Microsurgery. 1989; 10:220-225. [PubMed: 2796718]

16. Barth TM, Jones TA, Schallert T. Functional subdivisions of the rat somatic sensorimotor cortex. Behav Brain Res. 1990; 39:73-95. [PubMed: 2390194]

17. O’Brien, BM., Morrison, WA. Reconstructive Microsurgery. Edinburgh, NY: Churchill Livingstone; 1987. Nerve grafts.

18. Wolford LM, Stevao EL. Considerations in nerve repair. Proc (Bayl Univ Med Cent). 2003; 16:152-156. [PubMed: 16278731]

19. Sunderland, S. Nerve Injuries and Their Repair. Edinburgh, NY: Churchill Livingstone; 1992. Nerve grafting and related methods of nerve repair.

20. Yamasaki T, Fujiwara H, Oda R, et al. In vivo evaluation of rabbit sciatic nerve regeneration with diffusion tensor imaging (DTI): correlations with histology and behavior. Magn Reson Imaging. 2015; 33:95-101. [PubMed: 25271136]

21. Stanisz GJ, Midha R, Munro CA, et al. MR properties of rat sciatic nerve following trauma. Magn Reson Med. 2001; 45:415-420. [PubMed: 11241698]

22. Morisaki S, Kawai Y, Umeda M, et al. In vivo assessment of peripheral nerve regeneration by diffusion tensor imaging. J Magn Reson Imaging. 2011; 33:535-542. [PubMed: 21287654]

23. Jambawalikar S, Baum J, Button T, et al. Diffusion tensor imaging of peripheral nerves. Skeletal Radiol. 2010; 39:1073-1079. [PubMed: 20593175]

24. Takagi T, Nakamura M, Yamada M, et al. Visualization of peripheral nerve degeneration and regeneration: monitoring with diffusion tensor tractography. Neuroimage. 2009; 44:884-892. [PubMed: 18948210]

25. Brenner MJ, Moradzadeh A, Myckatyn TM, et al. Role of timing in assessment of nerve regeneration. Microsurgery. 2008; 28:265-272. [PubMed: 18381659] 

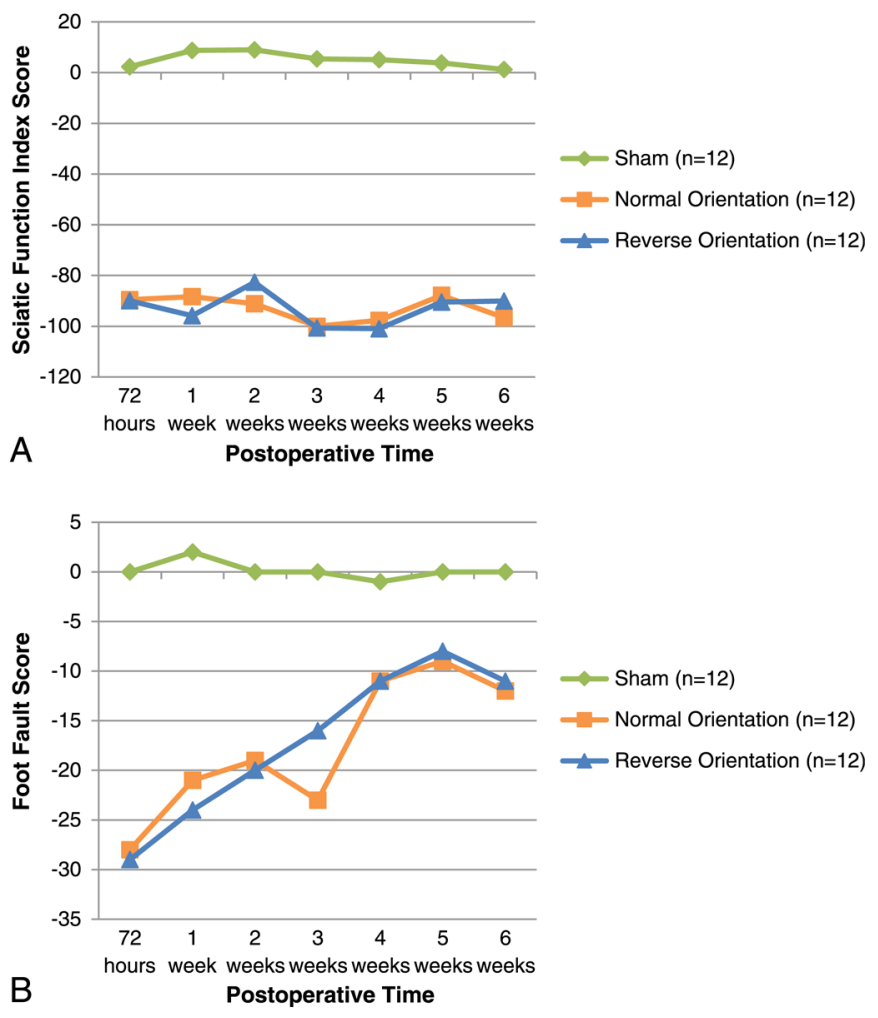

FIGURE 1.

A, Sciatic function index scores for shams, normal, and reverse orientated autografts at 72 hours and weekly postoperative time points. A more negative score indicates more impairment. B, FF asymmetry scores for shams, normal, and reverse orientated autografts at 72 hours and weekly postoperative time points. A more negative score indicates more impairment. 


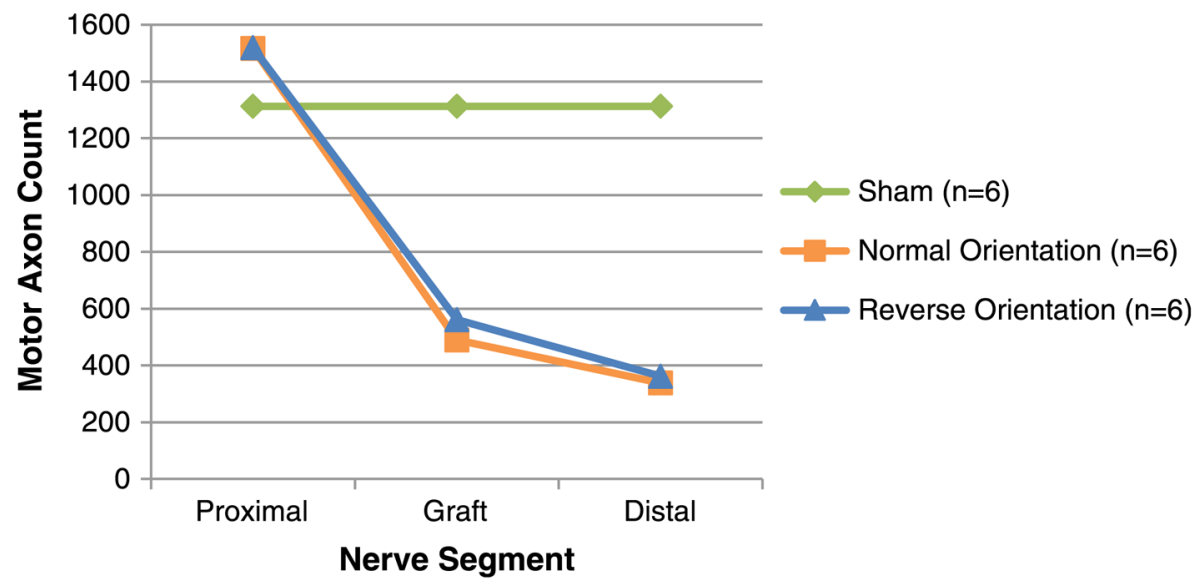

FIGURE 2.

Motor axon counts using immunohistochemistry at low magnification $(\times 10)$ for shams, normal, and reverse orientated autografts. Motor axon counts for normal and reverse oriented autografts were evaluated at segments proximal, within, and distal to the graft site, whereas only at 1 segment for shams. 


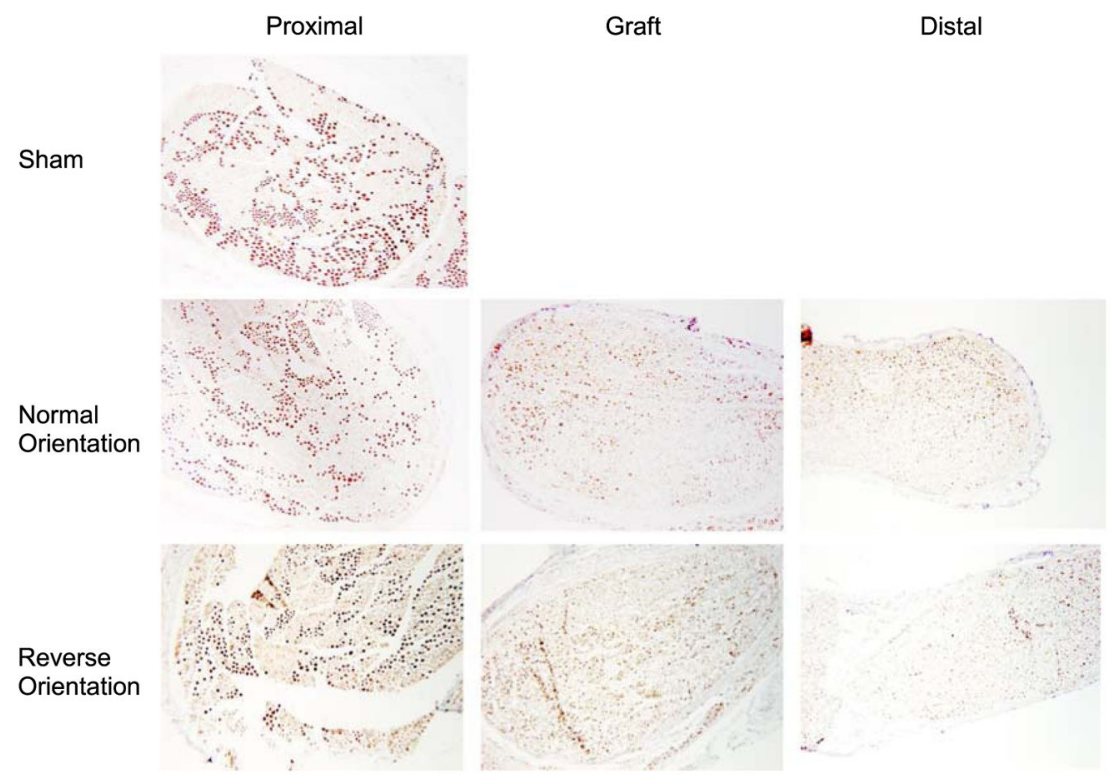

FIGURE 3.

Representative images of immunohistochemical choline acetyltransferase stain for motor axons at low magnification $(\times 10)$ for shams, normal, and reverse orientated autografts. Motor axons for normal and reverse oriented autografts were evaluated at segments proximal, within, and distal to the graft site, whereas only at 1 segment for shams as a reference. No difference was observed in motor axon count between normal and reverse oriented autografts. 

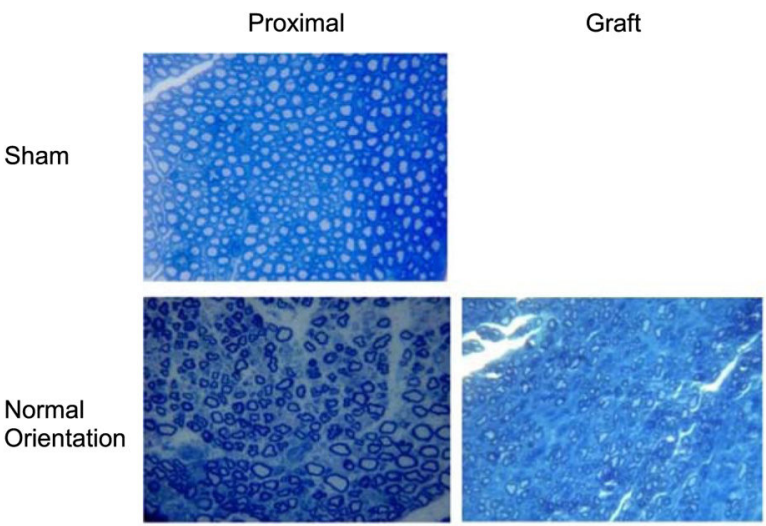

Distal
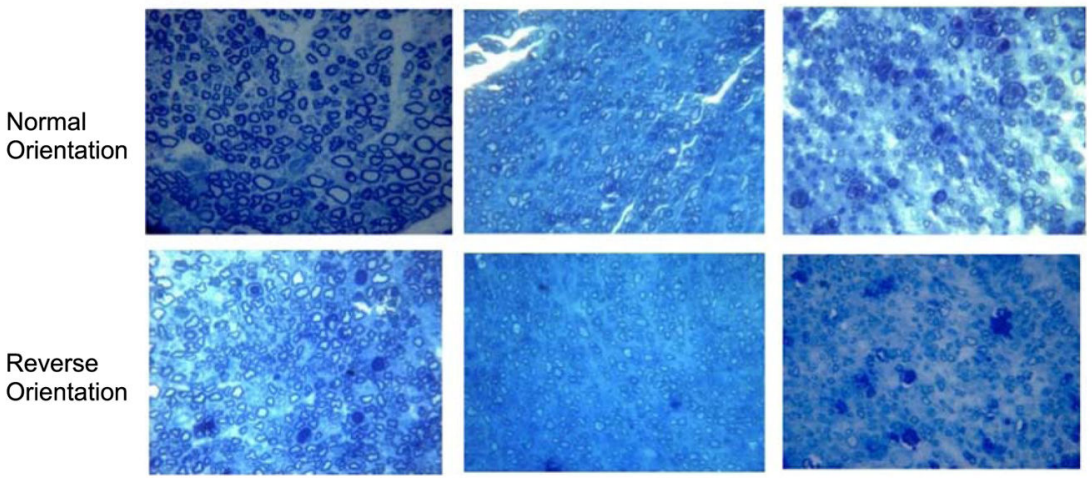

FIGURE 4.

Representative images of toluidine blue axon stains of $1-\mu \mathrm{m}$-thick section at $\times 40$ magnification for shams, normal, and reverse orientated autografts. Axons for normal and reverse oriented autografts were evaluated at segments proximal, within, and distal to the graft site, whereas only at 1 segment for shams as a reference. No difference was observed in total axon count, axon density, or diameter between normal and reverse oriented autografts. 


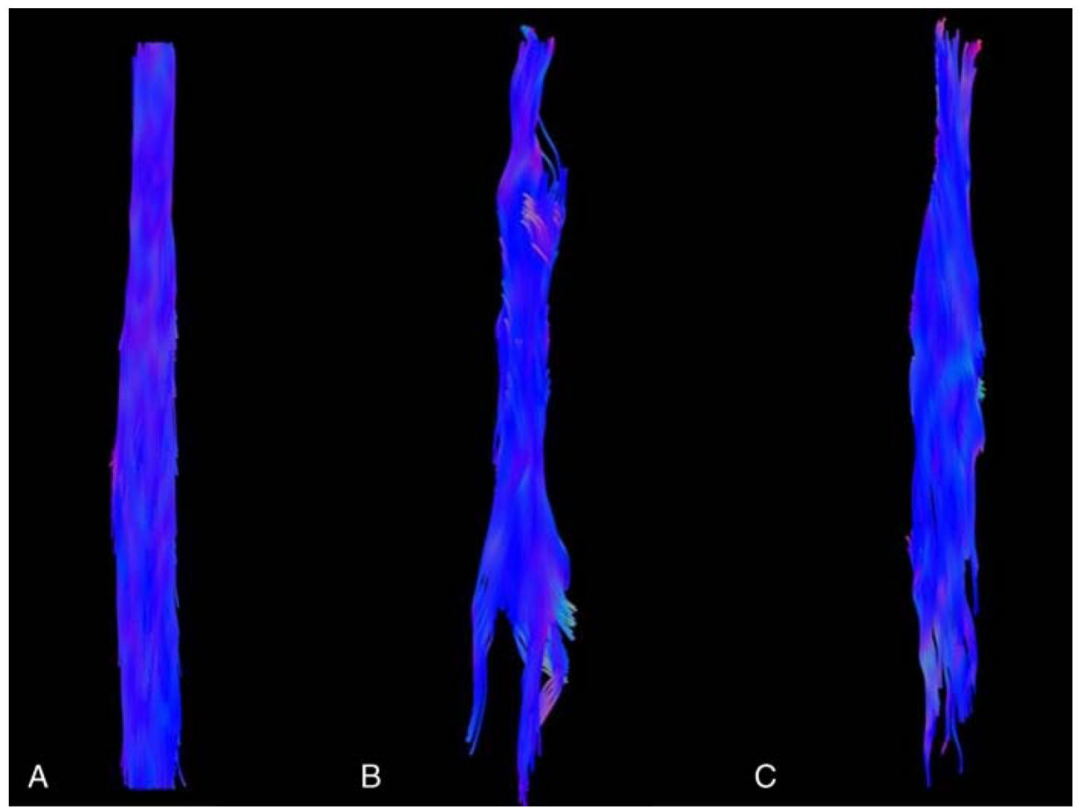

FIGURE 5.

Diffusion tensor imaging tractography images at 6 weeks demonstrating nerve track regeneration for (A) sham, (B) normal orientated, and (C) reverse orientated autografts. Normal and reverse autografts both demonstrate proximodistal regeneration, however with less linear directionality compared with shams. 


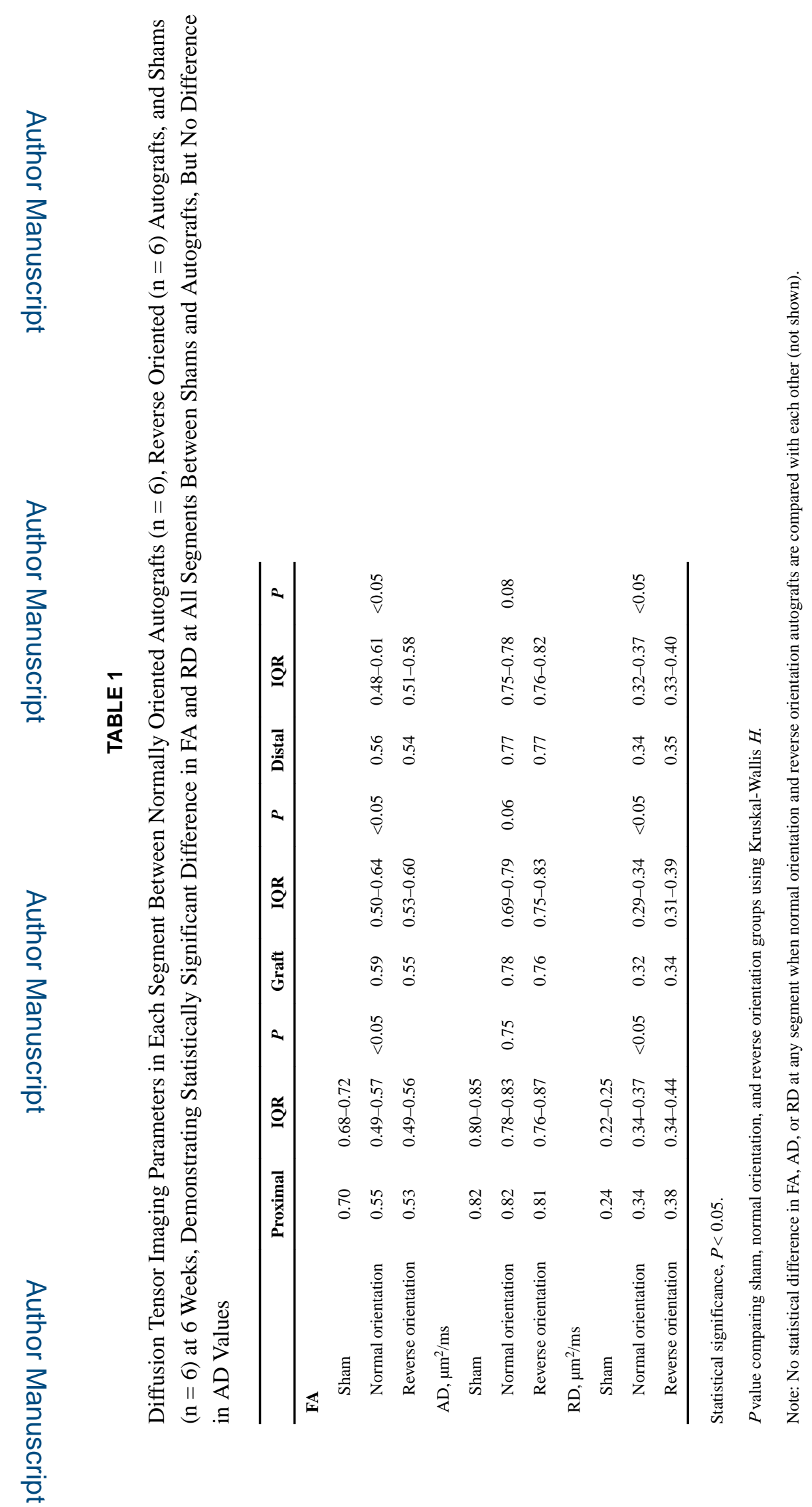

Ann Plast Surg. Author manuscript; available in PMC 2019 April 01. 